\title{
SD/Rij, Rat Strain
}

National Cancer Institute

\section{Source}

National Cancer Institute. SD/Rii, Rat Strain. NCI Thesaurus. Code C14381.

Less sensitive to the induction of mammary tumors by DMBA than two samples of outbred Sprague-Dawley rats; mean life-span 743-22 days. 